\title{
New Sierpenski Curves in Complex Plane
}

\author{
Priti Dimri \\ Associate Professor, \\ Department of Computer \\ Science and Engineering, \\ G.B Pant Engineering College, \\ Pauri Garhwal, 246001
}

\author{
Munish Singh Chauhan \\ Information Technology \\ Department \\ IBRI College of Applied \\ Sciences \\ Ministry of Higher Education, \\ Sultanate of Oman,
}

\author{
Ashish Negi \\ Associate Professor, \\ Department of Computer \\ Science and Engineering, \\ G.B Pant Engineering College \\ Pauri Garhwal, 246001
}

\begin{abstract}
The Sierpinski triangle also known as Sierpinski gasket is one of the most interesting and the simplest fractal shapes in existence. There are many different and easy ways to generate a Sierpinski triangle. In this paper we have presented a new algorithm for generating the sierpinski gasket using complex variables.
\end{abstract}

\section{Keywords}

Sierpinski Gasket, Fractal Coloring, Complex variables

\section{INTRODUCTION}

There is a lot of history behind Fractals even though the word fractal did not exist until the 1970s [1]. The Cantor Set discovered in 1872 by Georg Cantor [9], Sierpinski Gasket in 1916 by Wallow Sierpinski who created a triangle and a carpet, Koch Curves in 1904 by Helge Von Koch [10], Lévy C curve in 1938 by Paul Pierre Lévy have been known for quite some time [17].

Benoit Mandelbrot also known as the father of fractal geometry, discovered a fractal which was later known as the Mandelbrot Set (M-Set) [5]. According to Mandelbrot the turning point in fractal study occurred in 1979-1980 with his research of the Fatou-Julia theory of iteration [17]. Later Mandelbrot connected the mathematical monsters of old together into the category of fractal geometry [14].

Since then Fractals have been an area which is being used an applied in various areas like study of turbulence in flows, Biosensor interactions, spread of forest fires and epidemics, used in fractal image compression, to produce footage for films such as Dante's Peak and Star Trek II: The Wrath of Khan [4], used to identify cancerous tumors [4]. Further its representation visually has made it easier for the students to understand than Euclidean geometry.

A number of techniques have been developed to generate the fractal shapes and used to produce fascinating images. Two techniques namely Koch construction, and function iteration in the complex domain have been popularized by Mandelbrot's book, have gained popularity. The construction of Koch curves is a language-theoretic approach and is generated by a rewriting system defined in the domain of geometric shapes[10]. The method of function iteration analyzes the sequences of numbers $\left\{X_{n}\right\}$ generated by the formula $\quad x_{n+1}=f\left(x_{n}\right)$ where $\mathrm{f}$ is a complex function. The colour of each point represents how quickly the values reach the escape point. Often black is used to show values that fail to escape before the iteration limit, and gradually brighter colors are used for points that escape. This gives a visual representation of how many cycles were required before reaching the escape condition.
The Mandelbrot Set $\mu$ for the quadratic is defined as the collection of all c $\in \mathrm{C}$ (where $\mathrm{C}$ is the complex plane) for which the orbit of the point 0 is bounded, [14]

i.e.,

$$
\mu=\left\{c \in C:\left\{f^{(n)}(0)_{n=0}^{\infty} \text { is bounded }\right\}\right.
$$

The distance between a point and the origin may be no greater than 2 in order to be included in the Mandelbrot Set i.e.

"If $|\mathrm{c}|>2$ and $|\mathrm{z}| \geq|\mathrm{c}|$, then the orbit of $\mathrm{z}$ escapes to $\infty$."

The Mandelbrot set can also be generated by using the complex variables. Consider the complex z-plane. The real z are on the $\mathrm{x}$-axis while the imaginary are on the $\mathrm{y}$-axis.

So for any complex number

$$
\begin{aligned}
& z=x+y i \\
& z_{n}=x_{n}+y_{n} i \\
& z_{n+1}=z_{n}^{2}+c \text { where } c=a+i b \\
& z_{n+1}=\left(x_{n}+y_{n} i\right)^{2}+a+i b
\end{aligned}
$$

\section{SIERPINSKI GASKET}

In 1916, Sierpinski Gasket was introduced by Polish Mathematician Waclaw Sierpinski [21] who was a professor at Lvov and Warsaw. One of the moons craters is named after him. The name "Sierpinski gasket" was given by B. Mandelbrot [18]. Originally constructed as a curve, this is one of the basic examples of self-similar sets. Both the Sierpinski triangle and the Sierpinski carpet have equivalent repetitive tiling arrangement.

The basic concept of constructing a sierpinski fractal is to subdivide an equilateral triangle into four separate equilateral triangles, each of $1 / 4$ the initial size. The next triangles then can be again subdivided and so on for an infinite amount time[15].

Sierpinski gasket also known as the Sierpinski triangle is a set of points in the plane which remain if one carries out the process infinitely often. Each of the three parts in the kth step is a scaled down version in sierpinski gasket we can find copies of whole new at every point. The gasket is composed from small but exact copies of itself that's why it is called strictly self similar. It plays an important role in the theory of curves [21]. The Sierpinski carpet and Sierpinski gasket are both constructed by removal of open sets from a closed set, so they are closed. Thus they are closed, bounded subsets of Euclidean space, and hence compact. Both the Sierpinski carpet and Sierpinski gasket are path connected [13].

The Sierpinski triangle is one of the most famous fractals and the Hausdorff dimension and measure are the most important characteristics of a fractal sets. The Sierpinski triangle is 
defined by an iterated function system, which satisfies a technical condition called the open set condition [16].

Thus it follows from Hutchinson's Theorem [11] that the Hausdorff dimension of sierpinski gasket is equal to $\log 3 / \log 2$.

\section{Definition of Sierpinski Triangle [8]}

The Sierpinski Triangle $S$ is defined to be the intersection $\bigcap_{k=0}^{\infty} S_{k}$ where $S$ is a closed set containing all points inside $S_{\mathrm{k}}$ $(\mathrm{k} \geq 1)$ and on the boundary of an equilateral triangle, and is obtained by removing all points inside the medial triangles of each "basic triangle" of $\mathrm{S}_{\mathrm{k}-1}$

Fractals have been investigated for their visual qualities as art, their relationship to explain natural processes, music, medicine, and in mathematics [19]. Fractal Art is a subclass of two-dimensional visual art, and is in many respects similar to photography .Fractal art can be created easily by using many coloring algorithms like divergence algorithm, convergence algorithms decomposition algorithm, orbit trap etc [2].

In our paper we present an algorithm for generating the images of the complex Sierpinski gasket. By making slight changes in the variables of the formula and using coloring algorithm we get variants of the Sierpinski gasket which we have presented in our paper.

\section{ALGORITHM FOR COMPLEX \\ SERPINSKI GASKET}

initialize $\mathrm{z}=$ coordinate $(\mathrm{x}, \mathrm{y})$

initialize float $\mathrm{x}=\operatorname{real}(\mathrm{z})$, float $\mathrm{y}=\operatorname{imag}(\mathrm{z})$,

float $\mathrm{a}=\mathrm{pi} / 3$,

initialize float theta $=\tan (a)$, boolean bail $=$ false, int $\mathrm{i}=0$

loop:

if $\mathrm{i}=1$

if (theta*x + theta $<y)$ OR $\left(-\right.$ theta $^{*} \mathrm{x}+$ theta $\left.<\mathrm{y}\right)$

OR $(\mathrm{y}<0)$

endif

assign $-2 * z$ to $z$

assign $(z+1 i *$ theta $)$ to $z$

assign $\mathrm{z}$ - offset to $\mathrm{z}$

else

assign $\mathrm{z}+$ offset to $\mathrm{z}$

assign real $(\mathrm{z})$ to $\mathrm{x}$

assign $\operatorname{imag}(\mathrm{z})$ to $\mathrm{y}$

if $(-$ theta* $\mathrm{x}+$ theta $>=\mathrm{y})$ AND $($ theta* $\mathrm{x}+$ theta $>=\mathrm{y})$

AND $(y>=0)$

then assign value true to bail

elseif (theta* $\mathrm{x}+$ theta $<\mathrm{y}$ )

then assign $=2 * z+4-1 i *$ theta to $\mathrm{z}$

elseif (-theta*x + theta $<y$ )

then $\operatorname{assign} 2 * \mathrm{z}-4-1 \mathrm{i} *$ theta to $\mathrm{z}$

elseif $(\mathrm{y}<0)$

then $\operatorname{assign} 2 * \mathrm{z}+2+1 \mathrm{i} *$ theta to $\mathrm{z}$

endif

assign $\mathrm{z}$ - offset to $\mathrm{z}$

endif

endloop

\section{GEOMETRY OF COMPLEX SIERPINSKI GASKET}

The fractal generated by our method shows Koch curve like structure due to which we name it as Koch Sierpinski Gasket. We observe that by simply playing with the formula we can generate many variants of the Sierpinski gasket and that all the variants are composed from small but exact copies of itself. The variants of the Sierpinski gasket exhibit triangular similarity. The variants of the Sierpinski gasket also exhibit various kinds of transformations like congruence, rotational, translational, reflections, symmetry etc.[8]

We observe that Fig. 4 and Fig. 6 show the effect of transformation. These figures exhibit the property of reflection transformations. In Fig 9 we present a figure which is similar to a Sierpinski carpet except that instead of circles or square we have a triangle.

\section{CONCLUSIONS AND REMARKS}

In this paper we have presented a new algorithm to generate the images that gives a new aspect to the Sierpinski Gasket. We have applied the various coloring functions and schemes to the fractals and have generated some beautiful artistic variants of the Sierpinski gasket. We also observe that one of the resulting Sierpinski gasket looks like the Koch snowflakes.

This paper also gives a view that by simply playing with the formula we can generate many variants of the sierpinski gasket and that all the variants are composed from small but exact copies of itself.

\section{REFERENCES}

[1] Barnsley, M., 1988, "Fractals Everywhere (San Diego: Academic Press, Inc).

[2] Barrallo, J. and Jones, D., 1999, "Coloring algorithms for dynamical systems in the complex plane", ISAMA 99 Proceedings, 31-38.

[3] Barralo, J. and Sanchez, S., 2001, "Fractals and multi layer colouring algorithms", Bridges Conference Proceedings 2001, 89.

[4] Crownover Richard M., "Introduction to Fractals and Chaos" (Jones and Bartlett, Boston, 1995).

[5] Devaney, R. and Keen, L. (Eds.), 1989, "Chaos and Fractals: the Mathematics Behind the Computer Graphics", Proceedings of Symposia in Applied Mathematics vol. 39 (Providence: American Mathematical Society).

[6] Devaney, R. L.: "Chaos, Fractals and dynamics, Computer experiments in mathematics", Menlo Park, Addison - Wessley (1992).

[7] Falconer K. J., "Techniques in Fractal Geometry", Wiley, 1997.

[8] Falconer, K., 2003, "Fractal Geometry: Mathematical Foundations and Applications" (West Sussex: John Wiley \& Sons, Ltd).

[9] George Cantor "On the Power of Perfect Sets of Points in Classics on Fractals" (Westview Press, 2004) pp.11-23.

[10] H. Von Koch, "On a continuous curve without tangents constructible from elementary geometry", Classics on fractals (G. Edgar, ed.), Addison-Wesley, Reading, Massachusetts, 1993, pp. 25-45.

[11] Hutchinson, J. E., "Fractals and Self-similarity”, Indiana Univ. Math. J.,30(1981), 713-747.

[12] Hyburn G. T. W, "Topological characterization of the Sierpinski curve”, Fund. Math. 45 (1958), 320-324.

[13] Louwsma Joel , "Homeomorphism groups of the sierpinski carpet and sierpinski gasket" 
[14] Mandelbrot, Benoit B.,"The Fractal Geometry of Nature". New York: W.H. Freeman and Company, 1982.

[15] Milnor J. and Tan L.A., "Sierpinski Carpet as Julia set". Appendix F in Geometry and dynamics of quadratic rational maps, Experiment. Math. 2 (1993), 37-83.

[16] Milnor., "Dynamics in One Complex Variable", Vieweg, 1999

[17] Peitgen, H. O.; Jurgens, H.; Saupe, D.: "Chaos and Fractals", New frontiers of science, New York Springer,1992 984pp.

[18] Peitgen, Heinz-Otto, and Peter H. Richter,"The Beauty of Fractals". New York: Springer Verlag, 1986.

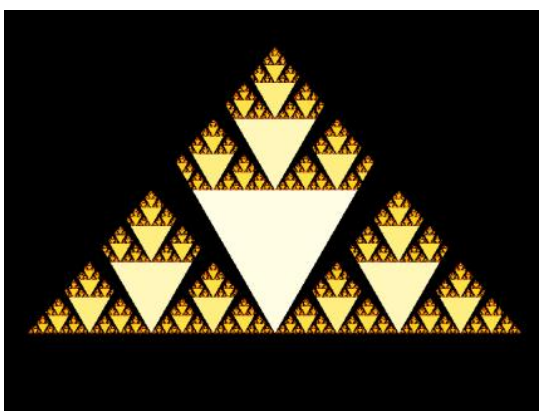

Fig 1. Koch Sierpinski Curve generated by our method

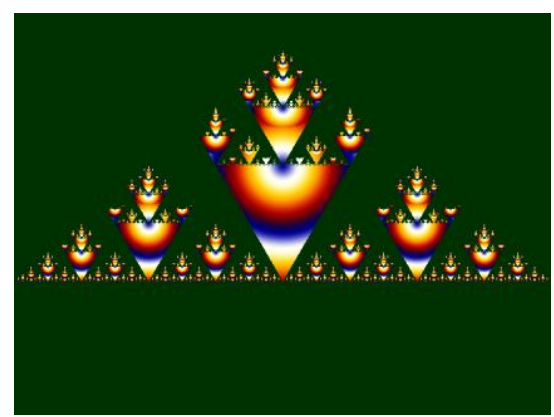

Fig 3: Figure obtained after applying Coloring algorithm to Fig 2.

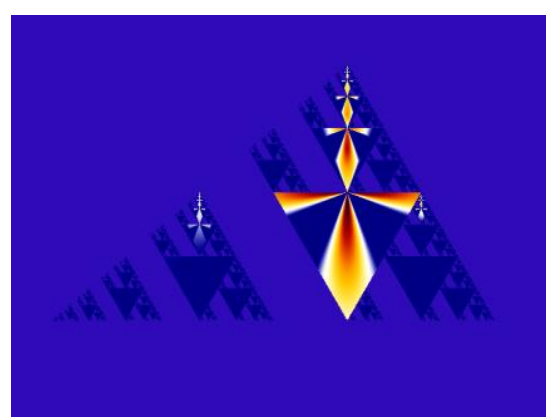

Fig 5.: Colouring algorithm applied to Fig 4.
[19] Pickover, C.A.," Computers, Pattern, Chaos,Beauty". St. Martin's Press. ISBN 0-486-41709-3

[20] Ponomarev S.P. "On some properties of Van Koch curves". Siberian Mathematical Journal, 2007, Vol. 48, No 6, 1046-1059 .]

[21] Warclaw Sierpinski, "Sur une courbe dont tout point est un point de ramication". Compt. Rendus Acad. Sci. Paris, 160:302\{305, 1915.

[22] Wegmann H., "Die HausdorfF-Dimension von kartesis"

[23] Weierstrass K., "On Continuous Functions of a Real Argument that do not have a Well-Defined Differential Quotient" in Classics on Fractals (Westview Press, 2004) pp.3-9.

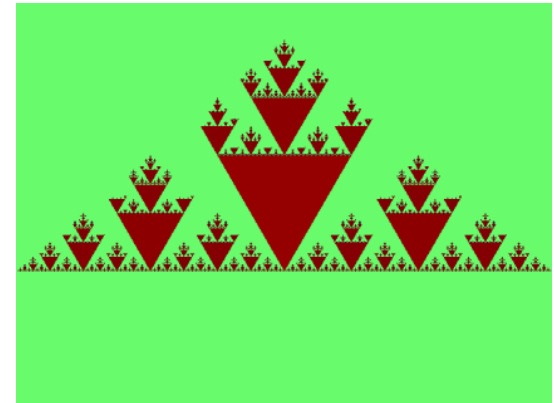

Fig 2: Koch sierpinski Curve with a different formula

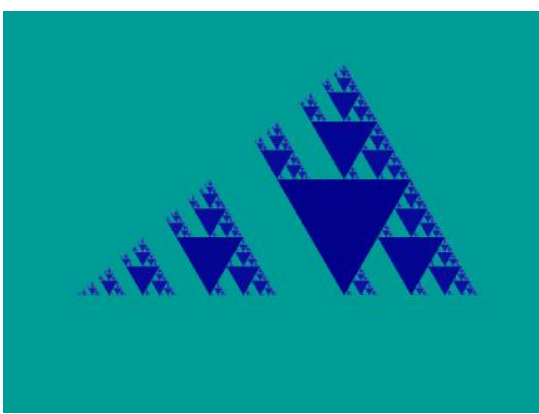

Fig 4. Sierpinski Gasket using a different variable in our algorithm

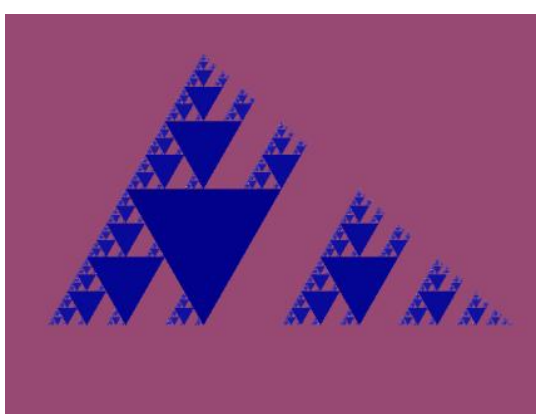

Fig6. Transformed version of Fig 4. 


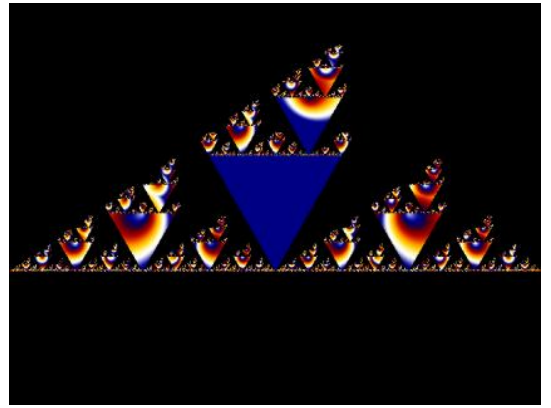

Fig 7: Figure obtained after application of Orbit Trap algorithm to our Formula.

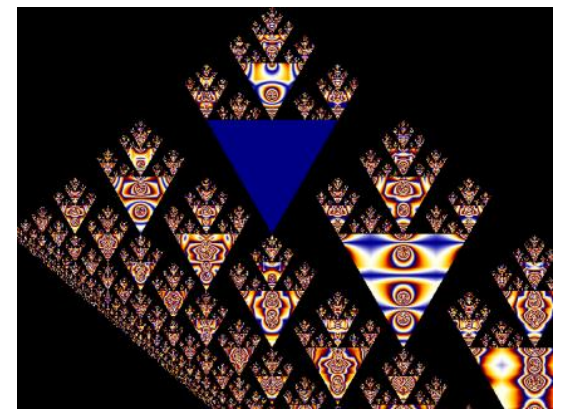

Fig 9. Left Elevated Triangular Sierpinski carpet

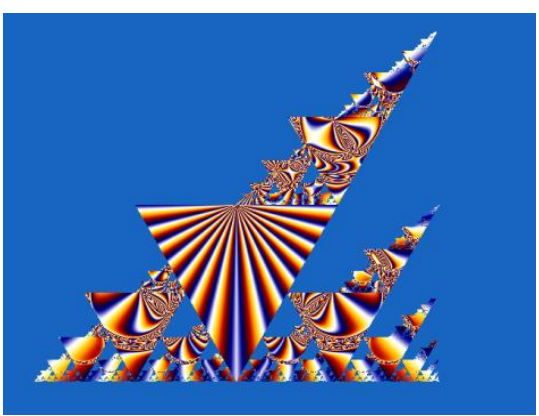

Fig 11: Right Elevated Koch Sierpinski Carpet

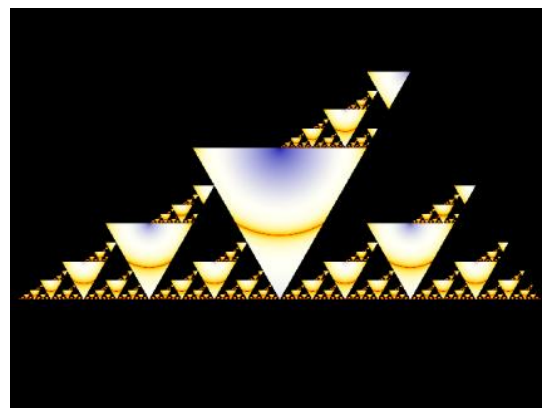

Fig 13.A different variant of our Sierpinski gasket

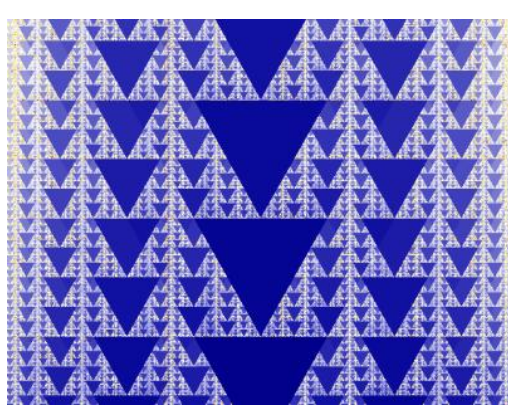

Fig 8. Triangular Sierpinski Carpet.

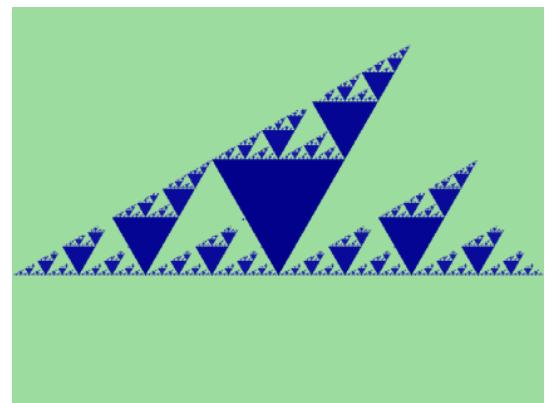

Fig 10. Triangular Sierpinski carpet using coloring algorithm

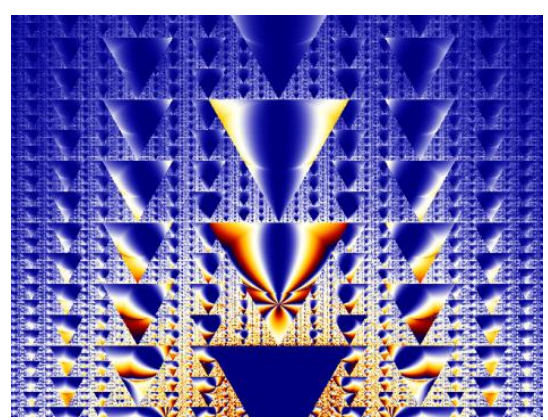

Fig 12: Orbit Trap Colouring Algorithm to Fig 8

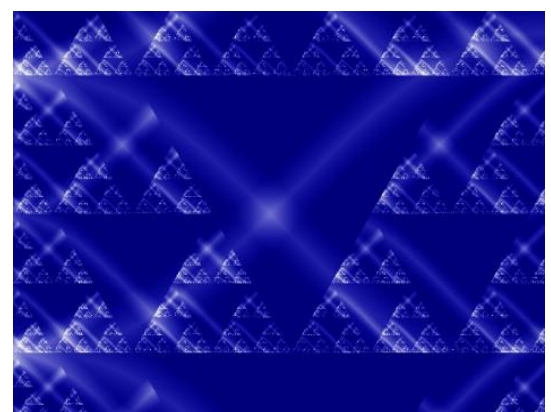

Fig14. Orbit Trap Colouring Algorithm to Fig 8 with orbit type=Point 